\title{
Disseminated central nervous system aspergillosis: A case report
}

\author{
Yimin Zhang ${ }^{1}$, Huiying Qiu ${ }^{1}$, Mingyuan Liu ${ }^{2}$, Lili Xu ${ }^{1}$, Xiaoxia Hu ${ }^{1 *}$, Jianmin Wang ${ }^{1 *}$ \\ ${ }^{\prime}$ Department of Hematology, Institute of Hematology, Changhai Hospital, Shanghai 200433, China; \\ ${ }^{2}$ Department of Neurology, Changhai Hospital, Shanghai 200433, China
}

Keywords: acute myeloid leukemia, aspergillus, central nervous system, hematopoietic stem cell transplantation

\section{INTRODUCTION}

Fungal infections of the central nervous system (CNS) are rare, but when present pose a life-threatening challenge ${ }^{[1]}$. Their prevalence spans a wide array of patient conditions, including immunosuppressed and immunocompetent individuals, patients undergoing neurosurgical procedures and those carrying implanted CNS devices. Cryptococcus neoformans and Aspergillus spp. remain the most common pathogens ${ }^{[2]}$. Patients with hematologic malignancy and neutropenia are particularly susceptible to CNS aspergillosis ${ }^{[3]}$. A clinically rare case of a patient with disseminated aspergillus lesions in the brain, after hematopoietic stem cell transplantation (HSCT) is presented in this report.

\section{CASE REPORT}

A 17-year-old young man was diagnosed with granuloma/acute myeloid leukemia with $\mathrm{T}$ lineage expression in November 2015. Presenting with multiple lines of chemotherapy, the disease was evaluated as being in progression. In May 2017, the patient received a salvage haplo-matched hematopoietic stem cell transplantation from his father. At 1 day after transplantation, the patient remained listless but without fever or headache. Upon neurological examination, he was confused and answered questions slowly,

*Correspondence to: Jianmin Wang, M.D, Department of Hematology, Changhai Hospital, 168 Changhai Road, Shanghai 200433, China. Tel/Fax: 86-213116-1293, E-mail: jmwangch@139.com; Xiaoxia Hu, M.D. Department of but had no stiffness of the neck, cranial-nerve palsies, pyramidal signs, or pupil changes. His lumbar puncture opening pressure was $145 \mathrm{cmH}_{2} \mathrm{O}$, while cerebrospinal fluid (CSF) analysis revealed pleocytosis (100 white cells per cubic millimeter, $90 \%$ segmented neutrocytes), elevated protein levels $(0.54 \mathrm{~g} / \mathrm{L})$ and low glucose levels $(2.2 \mathrm{mmol} / \mathrm{L})$. Magnetic resonance imaging(MRI) demonstrated multiple intracranial, round-like lesions of different sizes with low TIW signal (Fig. 1) and high T2W signal(Fig.2). Most of them appeared as annular lesions with significant restriction of diffusion and mid marginal enhancement. These findings were highly suggestive of invasive fungal infection, but may also be observed in patients with cryptococcal meningitis and encephalopyosis. No microorganisms were detected in the Gram staining of the CSF, and the latex-agglutination test for Cryptococcus neoformans of the CSF was negative. Acidfast bacilli were not seen on the staining of CSF, but galactomannan (GM) antigen using the PLATELIA ${ }^{\mathrm{TM}}$ assay were positive in both CSF and serum samples (11.5, 5.5, respectively, cut-off 0.5 ), leading us to treat the illness as an aspergillus infection. After the initiation of aggressive therapy with intravenously administered amphotericin B, the patient displayed a slowly deteriorating mental status, followed by seizure leading to cardiac failure. The patient died on the $8^{\text {th }}$ day post transplantation. Permission for a postmortem examination was not granted.

Hematology, Changhai Hospital, 168 Changhai Road, Shanghai 200433, China. Tel/Fax: 86-21-3116-1293, E-mail: huxiaoxia@medmail.com.cn. Conflict-of-interest: The authors bave declared no confliet of interests. 


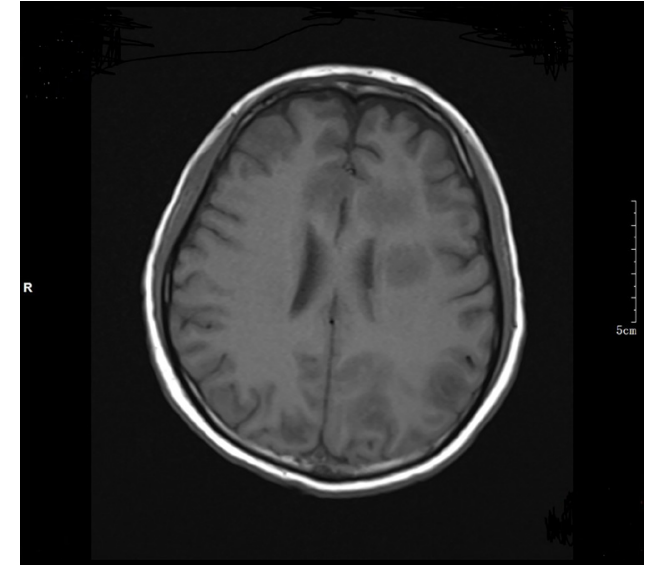

Fig. 1 Magnetic Resonance Imaging test of the patient's brain on the $+1 \mathrm{~d}$ post hematopoietic stem cell transplantation with T1W signal

\section{DISCUSSION}

Invasive fungal disease (IFD) is a serious complication after allogeneic stem cell transplantation (alloHSCT) with incidence rates ranging from $3 \%$ to $23 \%{ }^{[4]}$. Aspergillus is the most common pathogen, which accounts for more than $90 \%$ of pathogens, and leads to an extremely high mortality rate of $90 \% \sim 100 \%{ }^{[4]}$. Lungs and the sinuses are the most common sites directly involved, followed by other sites such as the central nervous system via dissemination ${ }^{[5]}$. The reported incidences of CNS involvement associated with invasive fungal infections are: invasive candidiasis $3 \% \sim 64 \%$, invasive aspergillosis $4 \% \sim 6 \%$, cryptococcosis $67 \% \sim 84 \%$, histoplasmosis (disseminated disease) $5 \% \sim 20 \%$, coccidioidomycosis (disseminated disease) $25 \%$, blastomycosis (patients with HIV/AIDS) $40 \%$ and zygomycosis $12 \%{ }^{[6]}$.

MRI can help locate the lesions, but diagnosis is challenging since the findings are non-specific. Other diagnostic techniques include the detection of GM or (1-3)-b-D-glucan in CSF or serum and molecular diagnostics with PCR-based techniques ${ }^{[7]}$.

This study reports a CNS invasive fungal disease in an allo-HSCT recipient. As in our case, fungal lesions are often multiple whereas bacterial abscesses are single, so a CSF positive result for GM is helpful in ascertaining fungal infection ${ }^{[5]}$. It is proved in a 3855-patient cohort (29 diagnosed with CNS-IFD) that those with pulmonary IFD were at higher risk of CNS-IFD, and the median onset time of CNS-IFD was 173 (24-972) days after the allo $\mathrm{HSCT}^{[4]}$. However, no signs of prior pulmonary infection were shown in our case. Moreover, our case showed a highly aggressive progression, with the

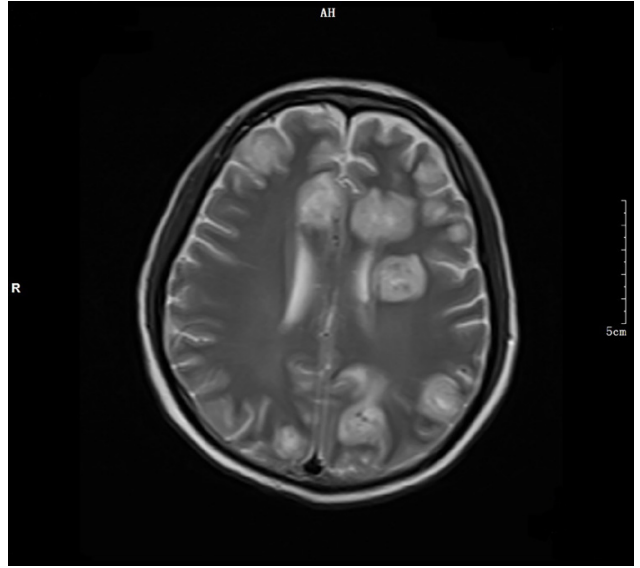

Fig. 2 Magnetic Resonance Imaging test of the patient's brain on the $+1 d$ post hematopoietic stem cell transplantation with T2W signal

patient died in a short period of time (only 8 days) with a poor response to the treatment of amphotericin B.

CNS-IFD is a rare but fatal complication after transplantation. Our case suggests that the identification of patients at high risk of CNS-IFD before HSCT is of significant importance. In our case, the risks were a poor responsive to prior treatment, neutropenia and systemic glucocorticoid treatment. The best choice of surgical methods, and/or antifungal drugs (including voriconazole or amphotericin B-or any effective combination based on the above) need to be investigated in further studies. Considering the high mortality rate, together with rapid progression, the diagnosis must be rapid, with effective medical and/or surgical intervention quickly implemented in order to successfully treat patients with CNS-IFD.

\section{Acknowledgments}

This work was supported by Municipal Human Resources Development Program for Outstanding Leaders in Medical Disciplines in Shanghai (2017BR012).

\section{References}

[1] Scully EP, Baden LR, Katz JT, et al. Fungal brain infections[J]. Curr Opin Neurol, 2008, 3(21): 347-352.

[2] Raparia K, Powell Z, Cernoch P, et al. Cerebral mycosis: 7-year retrospective series in a tertiary center[J]. Neuropathology, 2010, 3(30): 218-223.

[3] Kourbeti IS, Mylonakis E, Fungal central nervous system infections: prevalence and diagnosis[J]. Expert Rev Anti Infect Ther, 2014, 2(12): 265-273.

[4] Sun YQ, Liu ZY, Huang XJ, et al. A retrospective study of central nervous system invasive fungal disease after allogeneic stem cell transplantation: risk factors, clinical characteristics, and outcomes[J]. Biol Blood Marrow 
Transplant, 2017, 23(7): 1158-1164.

[5] McCarthy M, Rosengart A, Schuetz AN, et al. Mold infections of the central nervous system[J]. New Eng $J$ Med, 2014, 371: 150-160.

[6] Kethireddy S, Andes D, CNS pahrmacokinetics of antifungal agents[J]. Expert Opin Drug MetabToxicol, 2007, $3: 573-581$.
[7] Kourkoumpetis TK, Desalermos A, Muhammed M, et al. Central nervous system aspergillosis: a series of 14 cases from a general hospital and review of 123 cases from the literature[J]. Medicine, 2012, 6(91): 328-336.

(Received 08 March 2018, Revised 13 June 2018, Accepted 16 June 2018) 